PESQUIMAT 20 (1): 93-102 (2017)

ISSN:1560-912X/ ISSN-E:1609-8439

http://dx.doi.org/10.15381/pes.v20i1.13752

Facultad de Ciencias Matemáticas - UNMSM

\title{
Sobre el Teorema de Lyusternik en Espacios de Banach
}

\begin{abstract}
Milton Angelino Aycho Flores ${ }^{1}$, Tomás Alberto Núñez Lay ${ }^{2}$
Resumen: En este artículo se presenta el Teorema de Lyusternik, usado en problemas de optimización de funcionales definidas en espacios de Banach de cualquier dimensión, asimismo, en el estudio de la optimización con restricciones de funcionales Fréchet diferenciables bajo condiciones suficientemente regulares.
\end{abstract}

Palabras clave: Optimización; espacios de Banach; análisis funcional.

\section{About Lyusternik's Theorem in Banach Spaces}

\begin{abstract}
In this article we present to the Lyusternik theorem, used in optimization problems of functional defined in Banach spaces of any dimension, it is also, the study of constrained optimization Fréchet differentiable functional under sufficiently regular conditions.
\end{abstract}

Keywords: Optimization, Banach spaces, functional analysis.

Recibido: 20/12/2016. Aceptado:30/07/2017. Publicado online: 01/09/2017

(C)Los autores. Este artículo es publicado por la Revista PESQUIMAT de la Facultad de Ciencias Matemáticas, Universidad Nacional Mayor de San Marcos. Este es un artículo de acceso abierto, distribuido bajo los términos de la licencia Creative Commons Atribucion-No ComerciaCompartir Igual 4.0 Internacional.(http://creativecommons.org/licenses/by-nc-sa/4.0/) que permite el uso no comercial, distribución y reproducción en cualquier medio, siempre que la obra original sea debidamente citada. Para información, por favor póngase en contacto con revistapesquimat.matematica@unmsm.edu.pe

\footnotetext{
${ }^{1}$ UNMSM, Facultad de Ciencias Matemáticas. e-mail: maychof@unmsm.edu.pe

${ }^{2}$ UNMSM, Facultad de Ciencias Matemáticas, e-mail:tnunezl@unmsm.edu.pe
} 


\section{Introducción}

En la teoría de optimización clásica, dada una función diferenciable $f: D \subseteq \mathbb{R} \longrightarrow \mathbb{R}$, la condición necesaria para encontrar el punto óptimo de $f$ en $D$ es dar solución a la ecuación $f^{\prime}(x)=0$ donde $f^{\prime}(x)$ es la derivada de la función. Para el caso de una función real de varias variables reales, se utilizan los métodos del gradiente y la matriz Hessiana para resolver los problemas de optimización sin restricciones; asimismo el uso de la función Lagrangiana y los multiplicadores de Lagrange para el caso de la optimización con restricciones de igualdad.

Hacia la mitad del siglo XX, H. Kuhn y A. Tucker, estudian el problema de minimización de una función de varias variables, bajo restricciones o condiciones de desigualdad, obteniendo condiciones necesarias y suficientes para los puntos minimizantes. A partir de dichos resultados, muchos investigadores estudiaron la existencia de soluciones para el problema de minimización:

$$
\min _{x \in X} f(x),
$$

donde $X$ es un espacio normado y $f$ es un funcional real. La necesidad de esta extensión, se debe a que casi todos los modelos matemáticos de la vida real, en especial los modelos de aplicación hacia otras ramas de las ciencias exactas, trabajan sobre espacios que no son necesariamente el conjunto $\mathbb{R}$ o en general como el caso del espacio $\mathbb{R}^{n}$.

Para abordar el problema de la optimización en un espacio de Banach $X$, se emplean conceptos de diferenciabilidad más generales que la definición de derivada clásica, así como también resultados específicos del Análisis Convexo y del Análisis Funcional en dimensión infinita, para lograr la formulación del teorema de los multiplicadores de Lagrange y las condiciones de Kuhn-Tucker para funciones reales definidas en $X$.

En este artículo, se expone el teorema de Lyusternik [3] formulado en un espacio de Banach, el cual es considerado un resultado de vital importancia en la teoría de optimización abstracta, en la resolución problemas de optimización de funcionales sobre espacios normados, en particular en la optimización de funcionales sujeto a restricciones.

La versión original del teorema dada por Lyusternik en 1934, ha sido continuamente mejorada y generalizada por diversos autores. En Dmitruk [2] se presenta una generalización del teorema empleando métodos secuenciales denominados esquemas iterativos de Lyusternik consiguiendo resultados que permiten dar solución a optimización de funcionales no diferenciables. En el trabajo de Dontchev [3] se estudia una versión mejorada del teorema de la aplicación abierta para aplicaciones no lineales, bajo hipótesis de diferenciabilidad adecuadas. Como consecuencia de este resultado, se formula una nueva demostración de teorema de Lyusternik. En este artículo presentamos la demostración del teorema, siguiendo las ideas expuestas en Jahn [4] y Luenberger [5].

\section{Preliminares}

Considerar $X$ un espacio vectorial normado (e.v.n) con norma \|\|$, X^{\star}$ su espacio dual topológico dotado con la norma

$$
\|f\|_{X^{\star}}=\sup _{x \neq 0} \frac{|f(x)|}{\|x\|_{X}}
$$


donde $f \in X^{\star}$. Asimismo, si $X$ e $Y$ son dos espacios normados, se define el espacio de las funcionales lineales continuas $L(X, Y)$ equipado con la norma

$$
\|f\|_{L(X, Y)}=\sup _{x \neq 0} \frac{\|f(x)\|_{Y}}{\|x\|_{X}}
$$

Los siguientes resultados pueden ser vistos en [1] y [6].

Teorema 2.1 Sea $X$ un e.v.n. Si $x \in X$ entonces existe $l \in X^{\star}$ tales que $\|l\|_{X^{\star}}=1$ y $l(x)=\|x\|$.

Demostración. Ver [1, pp. 19].

Teorema 2.2 (Aplicación abierta) Sean $\left(X,\|\|_{X}\right),\left(Y,\|\|_{Y}\right)$ dos espacios de Banach y $T \in L(X, Y)$ una aplicación sobreyectiva. Entonces $T$ es una aplicación abierta; es decir, para todo conjunto abierto $U \subseteq X, f(U)$ es abierto en $Y$.

Demostración. Ver [6, pp. 61].

Las siguientes definiciones serán empleadas a lo largo de este artículo.

Definición 1. Sean $\left(X,\|\|_{X}\right)$ e $\left(Y,\|\|_{Y}\right)$ dos espacios normados, $S \subseteq X$ un subconjunto abierto no vacío y $f: S \longrightarrow Y$ una aplicación. Se dice que $f$ es Fréchet diferenciable en $\bar{x} \in S$ si existe una aplicación lineal continua $f^{\prime}(\bar{x}): X \longrightarrow Y$ tal que

$$
\lim _{\|h\|_{X} \rightarrow 0} \frac{\left\|f(\bar{x}+h)-f(\bar{x})-f^{\prime}(\bar{x}) h\right\|_{Y}}{\|h\|_{X}}=0
$$

$f^{\prime}(\bar{x})$ será llamada la derivada de Frechet de $f$ en $\bar{x}$.

Definición 2. Sea $(X,\|\|)$ un e.v.n, $S \subseteq X$ no vacío y $\bar{x} \in \bar{S}$.

1. Diremos que $u \in X$ es un vector tangente a $S$ en $\bar{x}$ si existen dos sucesiones $\left(x_{n}\right)_{n \in \mathbb{N}} \subseteq S$ y $\left(\lambda_{n}\right)_{n \in \mathbb{N}} \subseteq \mathbb{R}^{+}$tales que $\bar{x}=\lim _{n \rightarrow \infty} x_{n} \mathrm{y} u=\lim _{n \rightarrow \infty} \lambda_{n}\left(x_{n}-\bar{x}\right)$.

2. El conjunto $T(S, \bar{x})=\{u \in X / u$ es vector tangente a $S$ en $\bar{x}\}$ será llamado cono tangente secuencial de Bouligand a $S$ en $\bar{x}$ o cono contenedor de $S$ en $\bar{x}$.

En principio, presentamos el siguiente teorema referido al cono tangente $T(S, \bar{x})$.

Teorema 2.3 Sean $\left(X,\|\cdot\|_{X}\right)$ y $\left(Z,\|\cdot\|_{Z}\right)$ dos e.v.n y $h: X \longrightarrow Z$ una aplicación. Consideremos el conjunto $S=\left\{x \in X / h(x)=0_{Z}\right\}$ y $\bar{x} \in S$. Si h es Frechet diferenciable en $\bar{x}$ entonces

$$
T(S, \bar{x}) \subseteq W
$$

donde $W=\left\{x \in X / h^{\prime}(\bar{x}) x=0_{Z}\right\}$.

Demostración. Sea $y \in T(S, \bar{x})$. Si $y=0_{X}$ entonces $y \in W$ pues $h^{\prime}(\bar{x})$ es una aplicación lineal. Supongamos que $y \neq 0_{X}$, por la Definición 2 existen dos sucesiones $\left(x_{n}\right) \subseteq S$ y $\left(\lambda_{n}\right) \subseteq \mathbb{R}^{+}$tales que $\bar{x}=\lim _{n \rightarrow \infty} x_{n}$ e $y=\lim _{n \rightarrow \infty} \lambda_{n}\left(x_{n}-\bar{x}\right)$. 
Como $h$ es Frechet diferenciable en $\bar{x}$ tenemos

$$
\begin{aligned}
h^{\prime}(\bar{x}) y & =h^{\prime}(\bar{x})\left\{\lim _{n \rightarrow \infty} \lambda_{n}\left(x_{n}-\bar{x}\right)\right\} \\
& =\lim _{n \rightarrow \infty} \lambda_{n} h^{\prime}(\bar{x})\left(x_{n}-\bar{x}\right) \\
& =\lim _{n \rightarrow \infty} \lambda_{n}\left\{h\left(x_{n}\right)-h(\bar{x})-\left(h\left(x_{n}\right)-h(\bar{x})-h^{\prime}(\bar{x})\left(x_{n}-\bar{x}\right)\right)\right\} \\
& =-\lim _{n \rightarrow \infty} \frac{\lambda_{n}\left\|x_{n}-\bar{x}\right\|_{X}\left\{h\left(x_{n}\right)-h(\bar{x})-h^{\prime}(\bar{x})\left(x_{n}-\bar{x}\right)\right\}}{\left\|x_{n}-\bar{x}\right\|_{X}}\left(x_{n}, \bar{x} \in S\right) \\
& =-\lim _{n \rightarrow \infty}\left\|y_{n}\right\|_{X} \frac{\left\{h\left(x_{n}\right)-h(\bar{x})-h^{\prime}(\bar{x})\left(x_{n}-\bar{x}\right)\right\}}{\left\|x_{n}-\bar{x}\right\|_{X}}=-\|y\|_{X}\left\{0_{Z}\right\}=0_{Z} .
\end{aligned}
$$

En consecuencia, $h^{\prime}(\bar{x}) y=0_{Z}$ por lo tanto $y \in W$, mostrando así el resultado.

\section{El Teorema Central}

Teorema 3.1 Sean $\left(X,\|\|_{X}\right)$ y $\left(Z,\|\|_{Z}\right)$ dos espacios de Banach reales y $h: X \longrightarrow Z$ una aplicación. Consideremos el conjunto factible $S=\left\{x \in X / h(x)=0_{Z}\right\}$ y el punto $\bar{x} \in S$. Supongamos que

(i) $h$ es Fréchet diferenciable en una vecindad de $\bar{x}$.

(ii) $h^{\prime}(\cdot)$ es continua en $\bar{x}$.

(iii) $h^{\prime}(\bar{x})$ es una aplicación sobreyectiva.

Entonces $W$ es un subconjunto de $T(S, \bar{x})$.

Demostración. Por hipótesis $(i i i)$ la aplicación $h^{\prime}(\bar{x})$ es lineal, continua y sobreyectiva. Así por el teorema de la aplicación abierta $h^{\prime}(\bar{x})$ es una funcional lineal abierta. Como $B\left(0_{X}, 1\right)$ es abierto en $X$ entonces $h^{\prime}(\bar{x})\left(B\left(0_{X}, 1\right)\right)$ es abierto en $Z$ por tanto existe $\rho_{1}>0$ tal que $B\left(0_{Z}, \rho_{1}\right) \subseteq h^{\prime}(\bar{x})\left(B\left(0_{X}, 1\right)\right)$ puesto que $0_{Z}$ es punto interior de $h^{\prime}(\bar{x})\left(B\left(0_{X}, 1\right)\right)$.

Afirmación 1. $h^{\prime}(\bar{x})\left(B\left(0_{X}, 1\right)\right)$ es acotado.

En efecto, sea $w \in h^{\prime}(\bar{x})\left(B\left(0_{X}, 1\right)\right)$, así existe $u \in B\left(0_{X}, 1\right)$ tal que $w=h^{\prime}(\bar{x}) u$. Como $h^{\prime}(\bar{x}) \in$ $L(X, Z)$, tenemos que

$$
\begin{aligned}
\|w\|_{Z} & =\left\|h^{\prime}(\bar{x}) u\right\|_{Z} \\
& \leq\left\|h^{\prime}(\bar{x})\right\|_{Z^{\star}}\|u\|_{X} \leq\left\|h^{\prime}(\bar{x})\right\|
\end{aligned}
$$

con lo cual $w \in B\left(0_{Z},\left\|h^{\prime}(\bar{x})\right\|_{Z^{\star}}\right)$, demostrando nuestra afirmación 1.

Consideremos el conjunto

$$
\Gamma=\left\{\rho>0 / B\left(0_{Z}, \rho\right) \subseteq h^{\prime}(\bar{x})\left(B\left(0_{X}, 1\right)\right)\right\} .
$$

Claramente, $\rho_{1} \in \Gamma$, así $\Gamma$ es no vacío y por la Afirmación 1 , acotado superiormente. Sea $\rho_{0}=\sup \Gamma$. Consideremos $\epsilon \in\left(0, \frac{\rho_{0}}{2}\right)$ arbitrario. Por la hipótesis $(i i)$ existe $\delta>0$ tal que

$$
\left\|h^{\prime}(\tilde{x})-h^{\prime}(\bar{x})\right\|_{L(X, Z)} \leq \epsilon
$$

para todo $\tilde{x} \in B(\bar{x}, 2 \delta)$. Tomemos $\tilde{x}_{1}, \tilde{x}_{2} \in B(\bar{x}, 2 \delta)$, como $h\left(\tilde{x}_{2}\right)-h\left(\tilde{x}_{1}\right)-h^{\prime}(\bar{x})\left(\tilde{x}_{2}-\tilde{x}_{1}\right) \in Z$ por el Teorema 2.1, existe $l \in Z^{\star}$ tal que $\|l\|_{Z^{\star}}=1 \mathrm{y}$

$$
l\left(h\left(\tilde{x}_{2}\right)-h\left(\tilde{x}_{1}\right)-h^{\prime}(\bar{x})\left(\tilde{x}_{2}-\tilde{x}_{1}\right)\right)=\left\|h\left(\tilde{x}_{2}\right)-h\left(\tilde{x}_{1}\right)-h^{\prime}(\bar{x})\left(\tilde{x}_{2}-\tilde{x}_{1}\right)\right\|_{Z} .
$$


Definimos la función

$$
\begin{aligned}
\varphi:[0,1] & \longrightarrow \mathbb{R} \\
t & \longmapsto \varphi(t)=l\left(h\left(\tilde{x}_{1}+t\left(\tilde{x}_{2}-\tilde{x}_{1}\right)-t h^{\prime}(\bar{x})\left(\tilde{x}_{2}-\tilde{x}_{1}\right)\right) .\right.
\end{aligned}
$$

Dado que $l \in Z^{\star}$ podemos escribir $\varphi(t)$ como

$$
\left.\varphi(t)=(l \circ h)\left(\tilde{x}_{1}+t\left(\tilde{x}_{2}-\tilde{x}_{1}\right)\right)-t\left(l \circ h^{\prime}(\bar{x})\right)\left(\tilde{x}_{2}-\tilde{x}_{1}\right)\right)
$$

para cada $t \in[0,1]$. Observemos que $\varphi(0)=l \circ h\left(\tilde{x}_{1}\right)$ y $\varphi(1)=l\left(h\left(\tilde{x}_{2}\right)-h^{\prime}(\bar{x})\left(\tilde{x}_{2}-\tilde{x}_{1}\right)\right)$. Por la convexidad de $B(\bar{x}, 2 \delta)$ tenemos que $\tilde{x}_{1}+t\left(\tilde{x}_{2}-\tilde{x}_{1}\right) \in B(\bar{x}, 2 \delta)$ para todo $t \in[0,1]$ y por hipótesis $h$ es Frechet diferenciable en una vecindad de $\bar{x}$. En consecuencia, la aplicación $\varphi$ es diferenciable en $(0,1)$ y por la regla de la cadena se obtiene

$$
\begin{aligned}
\varphi^{\prime}(t) & =l\left(h^{\prime}\left(\tilde{x}_{1}+t\left(\tilde{x}_{2}-\tilde{x}_{1}\right)\right)\left(\tilde{x}_{2}-\tilde{x}_{1}\right)-l\left(h^{\prime}(\bar{x})\left(\tilde{x}_{2}-\tilde{x}_{1}\right)\right.\right. \\
& =l\left(h^{\prime}\left(\tilde{x}_{1}+t\left(\tilde{x}_{2}-\tilde{x}_{1}\right)\right)\left(\tilde{x}_{2}-\tilde{x}_{1}\right)-h^{\prime}(\bar{x})\left(\tilde{x}_{2}-\tilde{x}_{1}\right)\right) .
\end{aligned}
$$

Luego, por el teorema del valor medio para derivadas, existe $\bar{t} \in(0,1)$ tal que $\varphi(1)-\varphi(0)=\varphi^{\prime}(\bar{t})$. Notemos que

$$
\varphi(1)-\varphi(0)=l\left(h\left(\tilde{x}_{2}\right)-h^{\prime}(\bar{x})\left(\tilde{x}_{2}-\tilde{x}_{1}\right)\right)-l \circ h\left(\tilde{x}_{1}\right) .
$$

Por lo tanto, de (2), (3) y (4) conseguimos la desigualdad

$$
\begin{aligned}
\left\|h\left(\tilde{x}_{2}\right)-h\left(\tilde{x}_{1}\right)-h^{\prime}(\bar{x})\left(\tilde{x}_{2}-\tilde{x}_{1}\right)\right\|_{Z} & =l\left(h\left(\tilde{x}_{2}\right)-h\left(\tilde{x}_{1}\right)-h^{\prime}(\bar{x})\left(\tilde{x}_{2}-\tilde{x}_{1}\right)\right) \\
& =\varphi(1)-\varphi(0) \\
& =\varphi^{\prime}(\bar{t}) \\
& =l\left(h^{\prime}\left(\tilde{x}_{1}+\bar{t}\left(\tilde{x}_{2}-\tilde{x}_{1}\right)\right)\left(\tilde{x}_{2}-\tilde{x}_{1}\right)-h^{\prime}(\bar{x})\left(\tilde{x}_{2}-\tilde{x}_{1}\right)\right) \\
& \leq\|l\|_{Z^{\star}}\left\|h^{\prime}\left(\tilde{x}_{1}+\bar{t}\left(\tilde{x}_{2}-\tilde{x}_{1}\right)\right)-h^{\prime}(\bar{x})\right\|_{L(X, Z)}\left\|\tilde{x}_{2}-\tilde{x}_{1}\right\|_{X} .
\end{aligned}
$$

De esta desigualdad, considerando que $\|l\|_{Z^{\star}}=1$ y $\tilde{x}_{1}+\bar{t}\left(\tilde{x}_{2}-\tilde{x}_{1}\right) \in B(\bar{x}, 2 \delta)$ logramos

$$
\left\|h\left(\tilde{x}_{2}\right)-h\left(\tilde{x}_{1}\right)-h^{\prime}(\bar{x})\left(\tilde{x}_{2}-\tilde{x}_{1}\right)\right\|_{Z} \leq \epsilon\left\|\tilde{x}_{2}-\tilde{x}_{1}\right\|_{X}
$$

para cualesquiera $\tilde{x}_{1}, \tilde{x}_{2} \in B(\bar{x}, 2 \delta)$. Por la elección de $\epsilon$ tenemos que $\frac{\epsilon}{\rho_{0}}<\frac{1}{2}$. Tomemos $\alpha>1$ tal que $\alpha\left(\frac{\epsilon}{\rho_{0}}+\frac{1}{2}\right)<1$ y consideremos $x \in W$. Si $x=0_{X}$ el teorema se satisface puesto que $0_{X} \in T(S, \bar{x})$. Consideremos el caso en que $x \neq 0_{X}$ y tomemos $\lambda \in(0, \hat{\lambda}]$ fijo pero arbitrario, donde $\hat{\lambda}=\frac{\delta}{\|x\|_{X}}$. Como $h^{\prime}(\bar{x}) X=Z$, podemos definir las sucesiones $\left(r_{n}\right)$ y $\left(u_{n}\right)$ de la siguiente manera:

Para $r_{1}=0_{X}$ existe $u_{1} \in X$ tal que $h^{\prime}(\bar{x}) u_{1}=h\left(\bar{x}+\lambda x+r_{1}\right)$. Definimos $r_{2}=r_{1}-u_{1}$, para este elemento, existe $u_{2} \in X$ tal que $h^{\prime}(\bar{x}) u_{2}=h\left(\bar{x}+\lambda x+r_{2}\right)$. Análogamente, para $r_{3}=r_{2}-u_{2}$ existe $u_{3} \in X$ tal que $h^{\prime}(\bar{x}) u_{3}=h\left(\bar{x}+\lambda x+r_{3}\right)$. En consecuencia, para cada $n \in \mathbb{N}$ se obtienen las sucesiones $\left(r_{n}\right)$ y $\left(u_{n}\right)$ que satisfacen

$$
h^{\prime}(\bar{x}) u_{n}=h\left(\bar{x}+\lambda x+r_{n}\right), r_{n+1} \quad=r_{n}-u_{n},
$$

Observemos por la definición de $\Gamma$ y $\alpha>1$ se satisface $B\left(0_{Z}, \frac{\rho_{0}}{\alpha}\right) \subseteq h^{\prime}(\bar{x})\left(B\left(0_{X}, 1\right)\right)$. 
Afirmación 2. $\left\|u_{n}\right\|_{X} \leq \frac{\alpha}{\rho_{0}}\left\|h\left(\bar{x}+\lambda x+r_{n}\right)\right\|_{Z}$ para todo $n \in \mathbb{N}$.

En efecto, si $u_{n}=0_{X}$ la desigualdad es trivial. Supongamos que $u_{n} \neq 0_{X}$ y definamos $w_{n}=\frac{u_{n}}{\left\|u_{n}\right\|_{X}}$.

De esta manera $w_{n} \in S\left(0_{X}, 1\right)$, siendo este conjunto la frontera de la bola unitaria en $X$. Así tenemos

$$
\begin{aligned}
B\left(0_{Z}, \frac{\rho_{0}}{\alpha}\right) & \subseteq B\left(0_{Z}, \rho_{0}\right) \\
& \subseteq h^{\prime}(\bar{x})\left(B\left(0_{X}, 1\right)\right) .
\end{aligned}
$$

De esta última inclusión, como $w_{n} \in S\left(0_{X}, 1\right)$ se obtiene la desigualdad

$$
\frac{\rho_{0}}{\alpha} \leq\left\|h^{\prime}(\bar{x}) \frac{u_{n}}{\left\|u_{n}\right\|_{X}}\right\|_{Z} .
$$

Por (6) y (7) se obtiene

$$
\begin{aligned}
\left\|u_{n}\right\| & \leq \frac{\alpha}{\rho_{0}}\left\|h^{\prime}(\bar{x}) u_{n}\right\|_{Z} \\
& =\frac{\alpha}{\rho_{0}}\left\|h\left(\bar{x}+\lambda x+r_{n}\right)\right\|_{Z} .
\end{aligned}
$$

Demostrando la Afirmación 2.

Definimos $d(\lambda)=\|h(\bar{x}+\lambda x)\|_{Z}$ y $q=\frac{\epsilon \alpha}{\rho_{0}}$. Por la elección de $\lambda$ obtenemos

$$
\begin{aligned}
\|\lambda x\|_{X} & =\lambda\|x\|_{X} \\
& \leq \hat{\lambda}\|x\|_{X} \\
& =\delta
\end{aligned}
$$

Por lo tanto $\|\lambda x\|_{X} \leq \delta$, en consecuencia $\|\bar{x}+\lambda x-\bar{x}\| \leq \delta<2 \delta$. Luego por las hipótesis sobre $x \mathrm{y}$ $\bar{x}\left(h(\bar{x})=0\right.$ y $\left.h^{\prime}(\bar{x}) x=0\right)$, la desigualdad anterior y $(5)$, logramos

$$
\begin{aligned}
d(\lambda) & =\left\|h(\bar{x}+\lambda x)-h(\bar{x})-h^{\prime}(\bar{x})(\lambda x)\right\|_{Z} \\
& \leq \epsilon\|\lambda x\|_{X} \leq \epsilon \delta .
\end{aligned}
$$

Como $\alpha>1$ tenemos $q=\frac{\epsilon \alpha}{\rho_{0}} \leq 1-\frac{\alpha}{2}<\frac{1}{2}$.

Afirmación 3. Se cumplen las siguientes desigualdades

(a) $\left\|r_{n}\right\|_{X} \leq \frac{\alpha}{\rho_{0}} d(\lambda)\left(\frac{1-q^{n-1}}{1-q}\right)$

(b) $\left\|h\left(\bar{x}+\lambda x+r_{n}\right)\right\|_{X} \leq d(\lambda) q^{n-1}$

(c) $\left\|u_{n}\right\|_{X} \leq \frac{\alpha}{\rho_{0}} d(\lambda) q^{n-1}$ para cualquier $n \in \mathbb{N}$.

En efecto, procediendo por inducción sobre $n$.

Para $n=1$ tenemos:

(a) Como $r_{1}=0_{X}$ tenemos $0=\left\|r_{1}\right\|_{X} \leq \frac{\alpha}{\rho_{0}} d(\lambda)(0)$.

(b) $\left\|h\left(\bar{x}+\lambda x+r_{1}\right)\right\|_{Z}=\|h(\bar{x}+\lambda x)\|_{Z}=d(\lambda)$ (por definición de $d(\lambda)$ ). 
(c) De la Afirmación 2 y (b) tenemos

$$
\begin{aligned}
\left\|u_{1}\right\|_{X} & \leq \frac{\alpha}{\rho_{0}}\left\|h\left(\bar{x}+\lambda x+r_{1}\right)\right\|_{Z} \\
& =\frac{\alpha}{\rho_{0}}\|h(\bar{x}+\lambda x)\|_{Z} \\
& =\frac{\alpha}{\rho_{0}} d(\lambda) .
\end{aligned}
$$

Supongamos válidas las tres afirmaciones para $n$ (hipótesis inductiva).

Para $n+1$ tenemos,

(a) Por la definición de $r_{n}$, la desigualdad triangular e hipótesis inductiva $(a)$ y $(c)$ conseguimos

$$
\begin{aligned}
\left\|r_{n+1}\right\|_{X} & =\left\|r_{n}-u_{n}\right\|_{X} \\
& \leq\left\|r_{n}\right\|_{X}+\left\|u_{n}\right\|_{X} \\
& \leq \frac{\alpha}{\rho_{0}} d(\lambda)\left(\frac{1-q^{n-1}}{1-q}\right)+\frac{\alpha}{\rho_{0}} d(\lambda) q^{n-1} \\
& =\frac{\alpha}{\rho_{0}} d(\lambda)\left(\frac{1-q^{n-1}}{1-q}+q^{n-1}\right)=\frac{\alpha}{\rho_{0}} d(\lambda)\left(\frac{1-q^{n}}{1-q}\right) .
\end{aligned}
$$

(b) Por hipótesis inductiva, la definición de $q$ y (8) tenemos

$$
\begin{aligned}
\left\|\lambda x+r_{n}\right\|_{X} & \leq\|\lambda x\|_{X}+\left\|r_{n}\right\|_{X} \\
& \leq \delta+\frac{\alpha}{\rho_{0}} d(\lambda)\left(\frac{1-q^{n-1}}{1-q}\right) \\
& \leq \delta+\frac{\alpha}{\rho_{0}}(\epsilon \delta)\left(\frac{1-q^{n-1}}{1-q}\right) \\
& \leq \delta+\frac{\delta q}{1-q}\left(1-q^{n-1}\right) \\
& \leq \delta\left(1+\frac{q}{1-q}\left\{1-q^{n-1}\right\}\right) \leq 2 \delta
\end{aligned}
$$

y

$$
\begin{aligned}
\left\|\lambda x+r_{n}-u_{n}\right\|_{X} & =\left\|\lambda x+r_{n+1}\right\|_{X} \\
& \leq\|\lambda x\|_{X}+\left\|r_{n+1}\right\|_{X} \\
& \leq \delta+\frac{\alpha}{\rho_{0}} d(\lambda)\left(\frac{1-q^{n}}{1-q}\right) \\
& =\delta\left(1+\frac{q}{1-q}\left\{1-q^{n-1}\right\}\right) \leq 2 \delta
\end{aligned}
$$

puesto que el producto $\frac{q}{1-q}\left\{1-q^{n-1}\right\}$ es menor que 1 y $q=\frac{\epsilon \alpha}{\rho_{0}}$.

Luego, por la definición de $u_{n}$ y la linealidad de $h^{\prime}(\bar{x})$ conseguimos

$$
\begin{aligned}
\left\|h\left(\bar{x}+\lambda x+r_{n+1}\right)\right\|_{Z} & =\left\|h\left(\bar{x}+\lambda x+r_{n}-u_{n}\right)\right\|_{Z} \\
& =\left\|h\left(\bar{x}+\lambda x+r_{n}\right)-h\left(\bar{x}+\lambda x+r_{n}\right)+h\left(\bar{x}+\lambda x+r_{n}-u_{n}\right)\right\|_{Z} \\
& =\left\|-h(\bar{x})\left(-u_{n}\right)-h\left(\bar{x}+\lambda x+r_{n}\right)+h\left(\bar{x}+\lambda x+r_{n}-u_{n}\right)\right\|_{Z} \\
& =\left\|h\left(\bar{x}+\lambda x+r_{n}-u_{n}\right)-h\left(\bar{x}+\lambda x+r_{n}\right)-h(\bar{x})\left(-u_{n}\right)\right\|_{Z} .
\end{aligned}
$$


Considerando $\tilde{x}_{2}=\bar{x}+\lambda x+r_{n}-u_{n}$ y $\tilde{x}_{1}=\bar{x}+\lambda x+r_{n}$, de (9) y (10) logramos

$$
\begin{aligned}
\tilde{x}_{2}-\tilde{x}_{1} & =\left(\bar{x}+\lambda x+r_{n}-u_{n}\right)-\left(\bar{x}+\lambda x+r_{n}\right)=-u_{n} \\
\left\|\tilde{x}_{2}-\bar{x}\right\|_{X} & =\left\|\left(\bar{x}+\lambda x+r_{n}-u_{n}\right)-\bar{x}\right\|_{X}=\left\|\lambda x+r_{n}-u_{n}\right\|_{X}<2 \delta \\
\left\|\tilde{x}_{1}-\bar{x}\right\|_{X} & =\left\|\lambda x+r_{n}\right\|_{X}=<2 \delta .
\end{aligned}
$$

De (12), concluimos que los puntos $\bar{x}+\lambda x+r_{n}-u_{n}$ y $\bar{x}+\lambda x+r_{n}$ pertenecen a $B(\bar{x}, 2 \delta)$. Luego de (10), (5) y la hipótesis inductiva (c) obtenemos

$$
\begin{aligned}
\left\|h\left(\bar{x}+\lambda x+r_{n+1}\right)\right\|_{Z} & \leq \epsilon\left\|-u_{n}\right\| . \\
& \leq \frac{\epsilon \alpha}{\rho_{0}} d(\lambda) q^{n-1}=d(\lambda) q^{n}
\end{aligned}
$$

(c) De (13), resulta que

$$
\begin{aligned}
\left\|u_{n+1}\right\|_{X} & \leq \frac{\alpha}{\rho_{0}}\left\|h\left(\bar{x}+\lambda x+r_{n+1}\right)\right\|_{Z} \\
& \leq \frac{\alpha}{\rho_{0}} d(\lambda) q^{n} .
\end{aligned}
$$

Quedando demostrada la Afirmación 3.

Afirmación 4. La sucesión $\left(r_{n}\right)$ es de Cauchy en $X$.

En efecto, como $r_{n+1}=r_{n}-u_{n}$ para $n, k \in \mathbb{N}$ tenemos

$$
\begin{aligned}
\left\|r_{n+k}-r_{n}\right\|_{X} & =\left\|r_{n+k-1}-u_{n+k-1}-r_{n}\right\|_{X} \\
& =\left\|r_{n+k-2}-u_{n+k-2}-u_{n+k-1}-r_{n}\right\|_{X} \\
& =\left\|r_{n}-u_{n}-u_{n+1}-u_{n+2}-\cdots-u_{n+k-2}-u_{n+k-1}-r_{n}\right\|_{X} \\
& =\left\|u_{n}+u_{n+1}+u_{n+2}+\cdots+u_{n+k-2}+u_{n+k-1}\right\|_{X} \\
& \leq\left\|u_{n}\right\|_{X}+\left\|u_{n+1}\right\|_{X}+\left\|u_{n+2}\right\|_{X}+\cdots+\left\|u_{n+k-2}\right\|_{X}+\left\|u_{n+k-1}\right\|_{X} .
\end{aligned}
$$

De la Afirmación 3 y la desigualdad (14) tenemos

$$
\begin{aligned}
\left\|r_{n+k}-r_{n}\right\|_{X} & \leq\left\|u_{n}\right\|_{X}+\left\|u_{n+1}\right\|_{X}+\cdots+\left\|u_{n+k-2}\right\|_{X}+\left\|u_{n+k-1}\right\|_{X} \\
& \leq \frac{\alpha}{\rho_{0}} d(\lambda) q^{n-1}+\frac{\alpha}{\rho_{0}} d(\lambda) q^{n}+\cdots+\frac{\alpha}{\rho_{0}} d(\lambda) q^{n+k-3}+\frac{\alpha}{\rho_{0}} d(\lambda) q^{n+k-2} \\
& =\frac{\alpha}{\rho_{0}} d(\lambda) q^{n-1}\left\{1+q+\cdots+q^{k-2}+q^{k-1}\right\} \\
& =\frac{\alpha}{\rho_{0}} d(\lambda) q^{n-1}\left\{\frac{1-q^{k}}{1-q}\right\}<\frac{\alpha}{\rho_{0}} d(\lambda) q^{n-1}\left\{\frac{1-q^{k}}{1-q}\right\}<\frac{\alpha}{\rho_{0}} d(\lambda) \frac{q^{n-1}}{1-q}
\end{aligned}
$$

puesto que $1-q^{k}<1$. Por lo tanto $\left\|r_{n+k}-r_{n}\right\|_{X}<\frac{\alpha}{\rho_{0}} d(\lambda) \frac{q^{n-1}}{1-q}$. Haciendo tender $n \rightarrow \infty$ el termino $q^{n-1}$ tiende a cero, en consecuencia $\left\|r_{n+k}-r_{n}\right\|_{X} \rightarrow 0$ si $n \rightarrow \infty$. De donde la sucesión $\left(r_{n}\right)$ es de Cauchy en $X$. Demostrando así la Afirmación 4.

Como $X$ es un espacio de Banach existe $r(\lambda) \in X$ tal que $\lim _{n \rightarrow \infty} r_{n}=r(\lambda)$. Observemos que dicho límite depende de $\lambda$. Asimismo, siendo $u_{n}=r_{n+1}-r_{n}$, la sucesión $\left(u_{n}\right)$ es convergente y por la 
Afirmación 3, logramos

$$
\begin{aligned}
\left\|u_{n}\right\|_{X} & \leq \frac{\alpha}{\rho_{0}} d(\lambda) q^{n-1} \\
& \leq \frac{\alpha}{\rho_{0}}(\epsilon \delta) q^{n-1} \\
& \leq \delta q^{n}
\end{aligned}
$$

Obteniendo la desigualdad $\left\|u_{n}\right\|_{X} \leq \delta q^{n}$. Tomando límite cuando $n \rightarrow \infty$ tenemos que $\left(u_{n}\right) \rightarrow 0_{X}$.

Afirmación 5. $\lim _{n \rightarrow \infty} \frac{r(\lambda)}{\lambda}=0_{X}$.

En efecto, como $h^{\prime}(\bar{x}) \in L(X, Z), u_{n} \longrightarrow 0_{X}, r_{n} \longrightarrow r(\lambda)$ y por la identidad (6) tenemos

$$
h(\bar{x}+\lambda x+r(\lambda))=h^{\prime}(\bar{x})\left(0_{X}\right)=0,
$$

puesto que $\bar{x}+\lambda x+r_{n} \in B(\bar{x}, 2 \delta)$. Por la Afirmación 3, obtenemos la desigualdad

$$
\left\|r_{n}\right\|_{X} \leq \frac{\alpha}{\rho_{0}} d(\lambda)\left(\frac{1-q^{n-1}}{1-q}\right)
$$

Tomando límite cuando $n \rightarrow \infty$ a la desigualdad anterior y dividiendo por $\lambda$ se obtiene

$$
\frac{\|r(\lambda)\|_{X}}{\lambda} \leq \frac{\alpha}{\lambda \rho_{0}} d(\lambda)\left(\frac{1}{1-q}\right)
$$

Por la definición de $d(\lambda)$, las igualdades $h(\bar{x})=0$ y $h^{\prime}(\bar{x}) x=0$ y efectuando la división por $\|x\|_{X}$, de (17) resulta que

$$
\begin{aligned}
\frac{\|r(\lambda)\|_{X}}{\lambda} & \leq \frac{\alpha}{\rho_{0}(1-q)} \frac{d(\lambda)}{\lambda} \\
& =\frac{\alpha}{\rho_{0}(1-q)} \frac{\left\|h(\bar{x}+\lambda x)-h(\bar{x})-\lambda h^{\prime}(\bar{x})(x)\right\|_{Z}\|x\|_{X}}{\lambda\|x\|_{X}} \\
& =\frac{\alpha}{\rho_{0}(1-q)} \frac{\left\|h(\bar{x}+\lambda x)-h(\bar{x})-h^{\prime}(\bar{x})(\lambda x)\right\|_{Z}}{\|\lambda x\|_{X}}\|x\|_{X} .
\end{aligned}
$$

Por otra parte, como $h$ es Frechet diferenciable en $\bar{x}$ tenemos que

$$
\lim _{\lambda \rightarrow 0^{+}} \frac{\left\|h(\bar{x}+\lambda x)-h(\bar{x})-h^{\prime}(\bar{x})(\lambda x)\right\|_{Z}}{\|\lambda x\|_{X}}=0 .
$$

En consecuencia, de (18) y (19) tenemos $\lim _{n \rightarrow \infty} \frac{\|r(\lambda)\|}{\lambda}=0_{X}$. Demostrando de esta manera la afirmación 5 .

Consideremos una sucesión $\left(\lambda_{n}\right) \subseteq \mathbb{R}^{+}$tal que $0<\lambda_{n}<\hat{\lambda}$ para todo $n \in \mathbb{N}$ y $\lim _{\lambda \rightarrow 0^{+}} \lambda_{n}=0$. Sean las sucesiones $\left(\mu_{n}\right) \subseteq \mathbb{R}^{+} \mathrm{y}\left(x_{n}\right) \subseteq X$ definidas por

$$
\mu_{n}=\frac{1}{\lambda_{n}} \quad y \quad x_{n}=\bar{x}+\lambda_{n} x+r\left(\lambda_{n}\right)
$$


para cada $n \in \mathbb{N}$, donde $r\left(\lambda_{n}\right)$ es el límite de la sucesión $\left(r_{k}\right)$ para el valor $\lambda_{n}$. Claramente $\mu_{n}>0$ y por $(6) h\left(x_{n}\right)=h\left(\bar{x}+\lambda_{n} x+r\left(\lambda_{n}\right)\right)=0_{Z}$. Así $x_{n} \in S$ para todo $n \in \mathbb{N}$. Por la afirmación 5 y la convergencia de $\left(\lambda_{n}\right)$ tenemos

$$
\begin{aligned}
\lim _{n \rightarrow \infty} x_{n} & =\lim _{n \rightarrow \infty} \bar{x}+\lambda_{n} x+r\left(\lambda_{n}\right) \\
& =\lim _{n \rightarrow \infty} \bar{x}+\lambda_{n}\left\{x+\frac{r\left(\lambda_{n}\right)}{\lambda_{n}}\right\} \\
& =\bar{x}
\end{aligned}
$$

y además

$$
\begin{aligned}
\lim _{n \rightarrow \infty} \mu_{n}\left(x_{n}-\bar{x}\right) & =\lim _{n \rightarrow \infty} \frac{1}{\mu_{n}}\left(\bar{x}+\lambda_{n} x+r\left(\lambda_{n}\right)-\bar{x}\right) \\
& =\lim _{n \rightarrow \infty} x+\frac{r\left(\lambda_{n}\right)}{\lambda_{n}}=x .
\end{aligned}
$$

Por lo tanto, $x \in T(S, \bar{x})$, es decir $W \subseteq T(S, \bar{x})$, quedando demostrado el teorema.

Observación. Del Teorema de Lyusternik y el teorema 3, cuando se satisfacen sus hipótesis respectivas, obtenemos la igualdad

$$
T(S, \bar{x})=W .
$$

\section{Conclusión}

El teorema de Lyusternik establece una relación entre el cono tangente y el núcleo de una aplicación suficientemente regular. Este resultado permite trabajar directamente con el cono tangente en problemas de optimización restringida cuando las funcionales de restricción cumplen ciertas hipótesis

de diferenciabilidad.

\section{Referencias bibliográficas}

[1] Barbu, V., Precupanu, T. (2012). Convexity and Optimization in Banach Spaces. 4ta. Ed. Dordrecht: Edit. Springer.

[2] Dmitruk, A., Milyutin, A., Osmolovskii, N. (1980). Lyusternik's Theorem and Theory of Extrema. Uspekhi Mat. Nauk, 35(6), 11-46.

[3] Dontchev, A. (1996) The Graves Theorem Revisited. Journal of Convex Analysis, 3(1), 45-53.

[4] Jahn, J. (2007). Introduction to the Theory of Nonlinear Optimization. 3era. Ed. Berlín: Edit. Springer.

[5] Luenberger, D. (1969). Optimization by Vector Spaces Methods. New York: Edit. John Wiley \& Sons.

[6] MacCluer, B. (2009). Elementary Functional Analysis. New York: Edit. Springer.

[7] Yu, G., Liu, S. (2007). Some Vector Optimization Problems in Banach Spaces with Generalized Convexity. Computers and Mathematics with Applications, 54, 1403-1410. 